\title{
Optimization of Scheduling for Small Batch and Customized Mixed-model Assembly Production
}

\author{
Xiao-ying YANG $^{1,2}$ \\ ${ }^{1}$ Advanced Machinery and Equipment Manufacturing \\ Collaborative Innovation Center in Henan Province, \\ Luoyang, China \\ ${ }^{2}$ School of Mechatronics Engineering, Henan University \\ of Science and Technology, Luoyang, China \\ E-mail:lyyxy111@163.com
}

\author{
Lin-ming $\mathrm{XU}^{2}$ \\ ${ }^{2}$ School of Mechatronics Engineering, Henan University \\ of Science and Technology, Luoyang, China \\ E-mail:1214402348@qq.com \\ Yu-zhe WANG ${ }^{3}$ \\ ${ }^{3}$ YTO Group Corporation, Luoyang, China \\ E-mail:lyyxy_haust@163.com
}

\begin{abstract}
In order to solve the complex problem of small batch and customized mixed-model assembly production (SBC-MAP) that lack of optimization scheduling, a optimization scheduling model and algorithm was studied by integrating the methods of math, management technology, information technology and a heuristic algorithm. Market fast response and balanced production were proposed as the target combining with market demand and production characteristics. The variation coefficient of delivery date and dispersion coefficient of balanced production were taken as the key performance indicator (KPI), and bottleneck materials and throughput were considered as the constraint. Eventually, the model of multiple objective and multiple constraint of scheduling optimization was constructed to realize the lean manufacturing goals. Meantime, to effectively solve the scheduling problem of fast and accurate, intelligent optimization algorithm was devised. The algorithm integrated online data acquisition, automatic Merge Sort (MS) and Adaptive Equalization (AE) algorithm, and search quality and efficiency were improved. Through practical application in agricultural machinery products showed the effectiveness and feasibility of the proposed model and algorithm.
\end{abstract}

Keywords: Small batch and customization, mixed-model assembly, scheduling, lean production, intelligent algorithm

\section{I.INTRODUCTION}

Small Batch and Customized Production (SBCP) as commonly production methods was widely used for the current enterprises to deal with fierce market competition, accommodate environmental changes and respond quickly to customers' individual needs. Compared with mass customization, the complexity of production management for SBCP is increasing quickly and its production optimization was considered as NP problem. In order to achieve low production costs, high quality and efficiency, products which have similar assembly process, but not the same type were assembled in one assembly line for Mixed-model Assembly Production (MAP). Mixed assembly production scheduling belongs to multiobjective optimization problems which according to the given optimization method and rule lists the assembly sequence in a certain period, it must be coincide with different customers order requirements of different models, different batches of products, and considering the delivery time, assembly process, material kitting, production line and other factors. The main feature of small batch and customized mixed-model assembly production (SBCMAP) is order stochastic uncertainties, multi variety and small batch, production instability, material change much and so on. In the current competitive market environment, the enterprise's issues of slow market response, difficulty of controlling the production process, material and delivery date was intensified due to lack effective scientific methods of scheduling. For solving those problems, scheduling optimization method for SBC-MAP needs to study through integration of information technology and management technique to provide scientific support.

\section{II.LITERATURE REVIEW}

The study of mixed-model assembly production derived from 1963 that Weste and Kilbridge [1] proposed scheduling problems of mixed-model assembly lines. Since then, this project has been studied by many scholars. They focused on the mixed-model assembly production scheduling optimization model and algorithm. In recent year, the representative achievements about scheduling optimization model: Maurizio Faccio[2] introduced an innovative balancing and sequencing hierarchical approach for paced mixed-model assembly lines using supplementary flexible workforce called 'jolly operators'. Hugejile Jo [3] studied the sort problem of automobile mixed-model assembly lines based on centralized assembling workload. Simon Emde[4] tackled the problem of determining when which part types should be taken from central receiving storage to the supermarket in what quantities. Ibrahim Kucukkoc[5] integrated the model sequencing problem in the line balancing procedure to obtain a more efficient solution for the problem of Simultaneous Balancing and Sequencing of Mixed-Model Parallel Two-Sided Assembly Lines and developed a 
mathematical model is developed to present the problem and a novel agent based ant colony optimization approach. ZümbülAtan[6] studied an assembly system with a number of parallel multistage processes feeding a multistage final assembly process and developed an iterative heuristic procedure based on this observation and a conjecture related to the generalized Newsvendor equations. LU Jian-sha[7] proposed a hybrid Artificial Bee Colony (ABC) algorithm with the objectives of minimizing total setup time, utility time and idle time and designed the neighborhood TS algorithm of bee colony. MENG Qiu-na[8] presented simultaneously minimize makespan, total time of earliness/tardiness and product conversion punishment as the objectives and proposed a improved multi - colony ant optimization algorithm to solving the model.NilsBoysen[9], LIU Qiong [10], Hsiang-Hsi Huang [11] studied the optimization model for mixed-model assembly line reordering. ZEMCZAK Marcin[12] presented the production orders sequencing issue, which main aim is to establish a proper sequence of tasks that would maximize the utilization of company's production capacity.

The main representative achievements about algorithms which solve the optimization problem of mixed-model assembly line schedule: Albert Corominas[13] discussed three points about the problem of sequencing units on a mixed-model assembly line in "A bit-wise mutation algorithm for mixed-model sequencing in JIT production systems. Yu-GuangZhong[14] considered two criteria to minimize the satisfaction ratio of delivery date and the complexity degree of the system arising from its state frequent changes and presented an improved particle swarm optimization (IPSO) algorithm to solve the multi-objective sequencing problems. Ibrahim Kucukkoc[15] addressed the problems of line balancing and model sequencing, and developed a novel hybrid agent-based ant colony optimization-genetic algorithm to solve the mixed model parallel two-sided assembly line balancing and sequencing problem. Jordi Pereira [16] developed a new branch-and-bound procedure for the problem that uses several new and previously proposed lower and upper bounds. F. Tonelli[17]analyzed and resolved some important issues related to technological, organizational and managerial constraints, and illustrated the implementation of an Advanced Planning System integrated with a mixed integer-programming model. In addition, improved harmony search (IHS) algorithm [18], Immunity Particle Swarm Optimization (PSO) [19], genetic algorithm (Haili Lu, [20], Wang Binggang, [21], HAN Yudong, [22], a new multiobjective dynamic fuzzy genetic algorithm (FarzadTahriri, [23]) are all the important achievements. The existing research results laid the foundation for this research, but most research was expansion based on medium Production, the achievement of SBC-MAP is uncommon.

Aiming at the lack research problem of SBC-MAP, a real-time optimization scheduling model and algorithm will be studied by integrating mathematics, management technology, information technology and a heuristic algorithm. Based on constraint conditions and optimization goal of lean production, the mathematical programming model for optimizing scheduling will be established. The optimization algorithm that integrate informationization and intelligence should be explored for solving the real-time, rapid analysis, and precise scheduling problem. The effectiveness and feasibility of the model and algorithm should be verified through practical application in agricultural machinery products. This study will provide an effective method that can improve market response speed and lean production level for manufacturer.

\section{III.PROBLEM MODEL AND OPTIMIZATION OBJECTIVE}

\section{A Problem Model}

SBC-MAP belongs to mode of multi variety and small batch production. The difficulty of this production mode is because that order instability leads to unbalance production, production resources conflict easily, production bottleneck-shifting, orders insert and orders cancel and so on. Present method is regard delivery date as the main measure, so as to meet customer's needs, but not considered lean production that results in low production efficiency and effectiveness.

This paper was guided by Lean Production (LP) thinking, production scheduling optimization not only considered to meet the delivery date, but also to guarantee production equilibrium in a certain period. We took production delivery order as first optimization goal, then the output and type equilibrium, eventually, achieve global optimum. Mixed assembly production scheduling belongs to multi-objective optimization problems, means that decompose complex optimization problems into optimization of variables, and ensures consistency between the variables through coordinate the sharing and coupling between variables by system constraints. The ultimate goal is global optimum for a system.

Problem Hypothesis: the value of $\mathrm{T}$ is planning period, $\mathrm{i}$ is product model, $T_{i}$ denotes the time of i model, $Q_{i}$ denotes the i model output, $q_{i t}$ denotes output of i model in $\mathrm{t}$ period. $Q_{i}=\left[q_{i 1}, \cdots, q_{i t}, \cdots, q_{i T}\right]$, the optimize space $\Omega=\left\{T_{i}, Q_{i}\right\}, \mathrm{F}\left(T_{i}, Q_{i}\right)$ is the objective function value in the status of $\left(T_{i}, Q_{i}\right)$. The demand is to search the optimum solution $\left(T_{i}, Q_{i}\right)^{*}$, so as to for all $\left(T_{i}, Q_{i}\right) \in \Omega$, $\mathrm{F}\left(T_{i}, Q_{i}\right)^{*}=\min \mathrm{F}\left(T_{i}, Q_{i}\right)$. Problem model as shown in figure 1 
Figure 1. Problem model of SBC-MAP

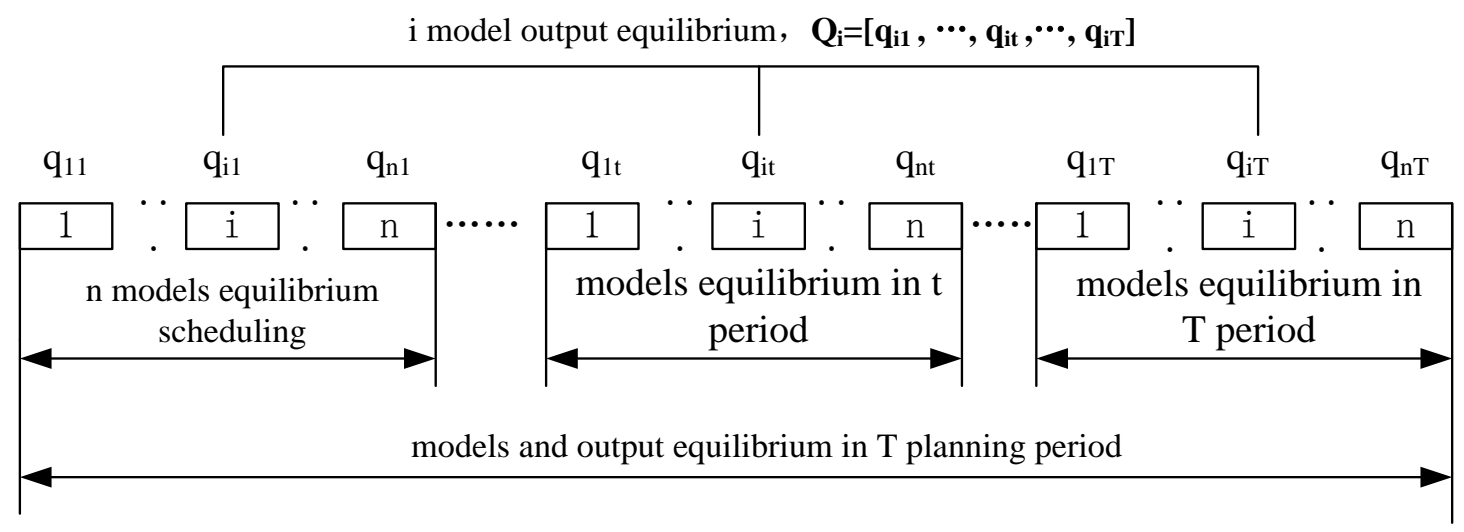

\section{B Optimization objective}

Optimization divided into two stages guided by lean production. Specific targets:

The first target is response speed for market: When manufacturing companies participate in market competition, the delivery speed that meets consumer requests is one of the most important factors for companies to obtain orders and improve core competence. These factors reflect the quick response ability to meet customer demand. This paper regards the minimum delivery response variance as the measure index. Presuming $S_{d}$ denotes the delivery response variance coefficient, $\mathrm{D}$ indicates delivery, $\mathrm{T}$ denotes the actual completion time, $\operatorname{so}_{d}=(D-T) / D$.

The second target is equilibrium production: product is many types, small batch and demand fluctuations. Equilibrium production within a year is difficult. Besides, the equilibrium of the labor load and rhythmic production need to be ensured, including daily yield equilibrium, daily assembly complexity equilibrium in a short term planning. This paper regards the minimum deviation coefficient as the equilibrium measure index, assuming $C_{v}$ denotes deviation coefficient, $\sigma$ denotes standard deviation, $\mu$ denotes mean value, $\operatorname{so}_{v}=\sigma / \mu$.

\section{IV.OPTIMIZATION MODEL}

\section{A Market response speed indicator: delivery response variance coefficient}

Presuming $\mathrm{n}$ denotes number of models, $\mathrm{i} \in \mathrm{n}, D_{i}$ denotes the delivery date request of model i, $T_{i}$ denotes completion time of scheduling, so the delivery response variance coefficient $S_{d}=\frac{1}{n} \sum_{i=1}^{n}\left(1-\frac{T_{i}}{D_{i}}\right)$.

\section{$B$ Balanced production indicator: dispersion coefficient}

Presuming $\mathrm{T}$ denotes planning period, $\mathrm{t} \in \mathrm{T}, q_{i t}$ denotes the $t$ days yield of model $i$, so the planned output of model i $Q_{i}=\left(q_{i 1}, q_{i 2}, \cdots, q_{i t}, \cdots, q_{i T}\right) . \mu$ is mean value, $\sigma$ is standard deviation, so the model complexity average of planning period $\mathrm{T}$ is $\mu_{i}=\frac{1}{T} \sum_{t=1}^{T} \sum_{i=1}^{n} i_{t} x, x=$ 0 or 1 , standard deviation $\sigma_{i}=\frac{1}{T} \sum_{t=1}^{T} \sum_{i=1}^{n}\left(i_{t} x-u_{i}\right)^{2}, x=$ 0 or 1 , daily yield $\quad \mu_{t}=\frac{1}{T} \sum_{t=1}^{T} \sum_{i=1}^{n} q_{i t}, \sigma_{t}=$ $\sqrt{\frac{1}{T} \sum_{t=1}^{T} \sum_{i=1}^{n}\left(q_{i t}-u_{t}\right)^{2}}$, model equilibrium deviation coefficient $C_{v_{i}}=\frac{v_{i}}{u_{i}}, \mathrm{i} \in \mathrm{n}$, daily yield equilibrium deviation coefficient $C_{v_{t}}=\frac{v_{t}}{u_{t}}, \mathrm{t} \in \mathrm{T}$.

Equilibrium production includes planned daily output equilibrium and model equilibrium. Assuming $\alpha$ denotes daily yield equilibrium weight, $\beta$ denotes model equilibrium, so total dispersion coefficient $C_{v}=\alpha C_{v_{i}}+$ $\beta C_{v_{t}}$, minimum deviation total $\operatorname{target} F\left(T_{i}, Q_{i t}\right)^{*}=C_{v}=$ $\alpha C_{v_{i}}+\beta C_{v_{t}}$.

Combining the objective of 4.1 and 4.2, the minimum total target $F\left(T_{i}, Q_{i t}\right)^{*}=\min \left[\gamma s d+\delta\left(\alpha C_{v_{i}}+\beta C_{v_{t}}\right)\right]$, $\gamma, \delta$ are two coefficients weight.

\section{$C$ Considering the constraints}

Firstly, considering the material constraints: assuming $\mathrm{k}$ denotes type of material, $t_{i k}$ denotes the $\mathrm{k}$ kind material expected time of arriving at line edge of model $i$, including material inventory, transit and delivery time, $t_{i}$ denotes the start time of assembling. The first step is to recognize bottleneck material when carrying out production scheduling, its condition: $t_{i k} \leq t_{i}$, otherwise, removing the model from production scheduling.

Secondly, considering capacity constraints: assuming Q denotes the daily maximum capacity, so the total yield of $\mathrm{t}$ days $Q_{t}=\sum_{i=1}^{n} q_{i t} \leq Q$.

\section{Establishment combinatorial optimization model}

According to the contents of 4.1, 4.2, 4.3, the combinatorial optimization model was established: 


$$
\begin{gathered}
\mathrm{S}_{\mathrm{d}}=\frac{1}{n} \sum_{i=1}^{n}\left(1-\frac{\mathrm{Ti}}{\mathrm{Di}}\right) \\
\mathrm{Cv}=\alpha \mathrm{Cvi}+\beta \mathrm{Cvt}=\alpha \frac{\sqrt{\frac{1}{T} \sum_{t=1}^{T} \sum_{i=1}^{n}\left(i_{t} x-\mathrm{u}_{\mathrm{i}}\right)^{2}}}{\frac{1}{T} \sum_{t=1}^{T} \sum_{i=1}^{n} i_{t} x}+\beta \frac{\sqrt{\frac{1}{T} \sum_{t=1}^{T} \sum_{i=1}^{n}\left(q_{i t}-\mathrm{u}_{\mathrm{t}}\right)^{2}}}{\frac{1}{T} \sum_{t=1}^{T} \sum_{i=1}^{n} q_{i t}}
\end{gathered}
$$

$\operatorname{Min} f\left(\mathrm{~T}_{\mathrm{i}}, \mathrm{q}_{\mathrm{it}}\right)=\gamma \mathrm{Sd}+\delta \mathrm{C}$

$$
\begin{aligned}
& =\gamma \frac{1}{n} \sum_{i=1}^{n}\left(1-\frac{\mathrm{Ti}}{\mathrm{Di}}\right)+\delta\left[\alpha \frac{\sqrt{\frac{1}{T} \sum_{t=1}^{T} \sum_{i=1}^{n}\left(i_{t} x-\mathrm{u}_{\mathrm{i}}\right)^{2}}}{\frac{1}{T} \sum_{t=1}^{T} \sum_{i=1}^{n} i_{t} x}+\beta \frac{\sqrt{\frac{1}{T} \sum_{t=1}^{T} \sum_{i=1}^{n}\left(q_{i t}-\mathrm{u}_{\mathrm{t}}\right)^{2}}}{\frac{1}{T} \sum_{t=1}^{T} \sum_{i=1}^{n} q_{i t}}\right] \\
& \text { s.t } t_{\mathrm{i} k} \leq \mathrm{t}_{i}, \quad \mathrm{i} \in \mathrm{n}, \quad \mathrm{k} \in \mathrm{K} \\
& \sum_{i=1}^{n} q_{i t} \leq Q, \quad \mathrm{i} \in \mathrm{n}, \quad \mathrm{t} \in \mathrm{T} \\
& \mathrm{x}=\left\{\begin{array}{rr}
0, & \text { model } i \text { isn't } \text { arrenged } \\
1, & \text { model } i \text { is arrenged }
\end{array}\right.
\end{aligned}
$$

$\mathrm{n}, \mathrm{T}, \mathrm{k}$ are all integer, $Q, q, t, \alpha, \beta, \varepsilon, \gamma \delta$ are all greater than 0 .

\section{V.OPTIMIZATION ALGORITHM BASED ON MES}

In order to avoid global blind search and low efficiency of traditional intelligent optimization algorithm, this article adopted heuristic optimization algorithm which integrated the intelligent scheduling and equilibrium optimization based on basic data, which including master production schedule (MPS), throughput, products and material of MES platform. Reducing search range and improving search efficiency through step by step optimization search is the primary characteristic of this algorithm.

Research ideas: according to the established optimization model, this article designed intelligent optimizing heuristic algorithm (IOHA) based on integrating MS use MES platform, AE and Java computer programming language. Eventually, artificial intelligence program based on empirical rules and optimization model was built. Phased search and online optimization by this artificial intelligence program, ultimately achieved global optimal, besides, this program is also convenient and efficient. MS is an automatic scheduling algorithm with high efficiency and good stability. AE algorithm is obtained the feasible solution procedure of adaptive function minimization which regards the optimization model as the adaptive function, constant search and project evaluation based on constraints. The design steps as shown in Figure 2.

Firstly, information acquisition and parameter setting: According to market plan, MPS, material requirement planning (MRP) were obtained from enterprise resource planning through MES. The data structure of MPS includes model coding, model, and demand quantity, demand delivery date, and get demand delivery date array, model coding array, demand quantity array. Setting up relevant parameter: daily maximum yield $\mathrm{Q}$, model number $\mathrm{n}$, material type $\mathrm{K}$ (material coding), per model require quantity $Q_{i}$, product coding $\mathrm{m}$, delivery requirement $D_{i}$, weight $\alpha, \beta, \gamma, \delta$, and Tzzk $\varepsilon$.

Secondly, using MS to schedule delivery requirement, $\mathrm{n}$ elements were divided into subsequence that contain $\mathrm{n} / 2$ elements, and then merge two subsequence, eventually discharge time series according to the urgency of delivery requirement (from small to large). If delivery requirement was same, then would sort the machine coding from small to large by using MS.

Thirdly, assuming the production scheduling is a matrix of two variables $\left[T_{i}, q_{i t}\right]$, sequential plan initial solution of $\mathrm{T}$ planned assembly production was discharged. In accordance of resource constraint $\sum_{i=1}^{n} q_{i t} \leq Q$, intelligent identification material status, all material status of daily production models were searched, checking $t_{i k} \leq t_{i}$, if this condition is not established, then remove and readjustment plan, and obtain production scheduling of per model daily yield array $\left[T_{i}, q_{i t}\right]$.

Fourthly, it is fit to compute fitness function $\mathrm{f}\left(T_{i}, q_{i t}\right)$ according to formula (3). The cyclic iteration $\mathrm{N}$ was started from step two, regarded the minimum value $\mathrm{f}\left(T_{i}, q_{i t}\right)$ as the feasible solution of sequential planning scheme. The solution is robust and reliable.

The flowchart of IOHA is showed in figure 2. This algorithm integrated online data acquisition, automatic MS and AE algorithm for improving search quality and efficiency, meanwhile, ensured the real-time and reliability of the scheduling scheme. 
Figure 2.Flowchart of IOHA

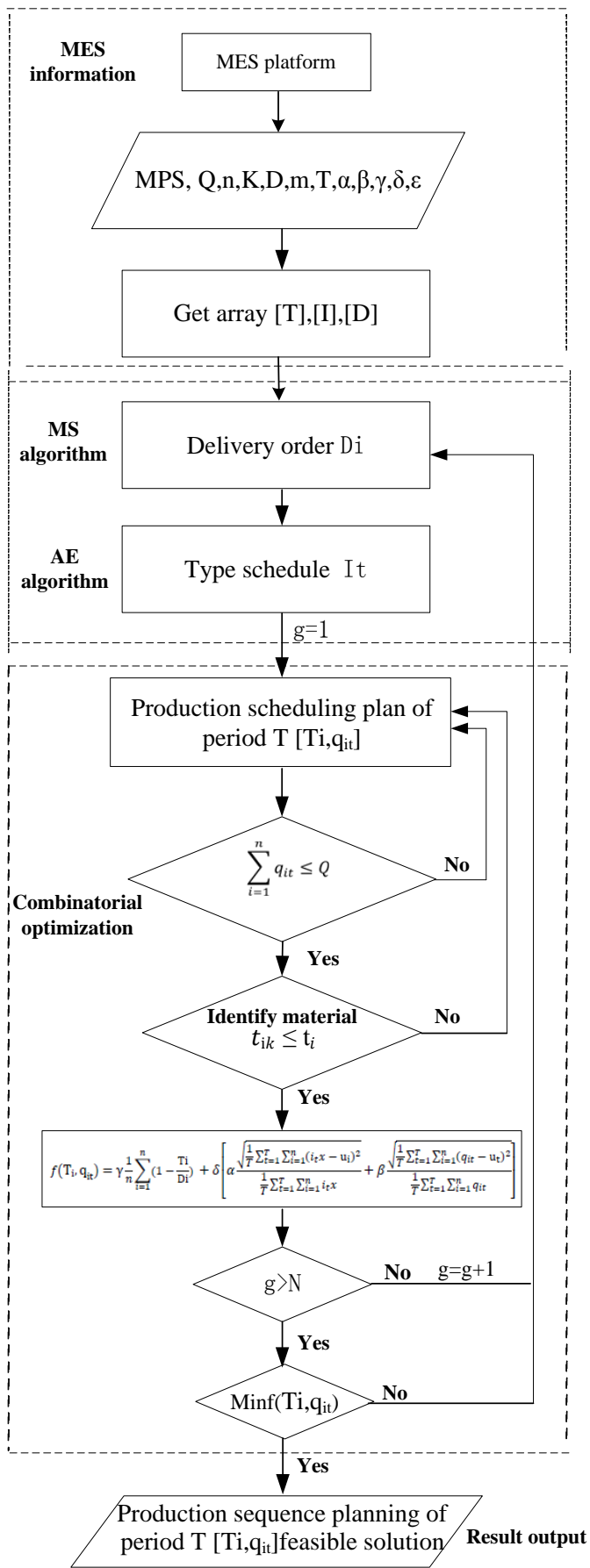

\section{VI.EXAMPLE APPLICATION}

\section{A Case description}

In this paper, tractor manufacturing was taken as an example to test the effectiveness of scheduling optimization model and algorithm. Tractor production is a typical SBC-MAP. The same tractor product series has the same structure, consists of basic component and some common parts, and there are several options for each basic component, so as to form a series of variety of deformation products. The general procedure of tractor production: The general procedure is inputs sales forecast plan into ERP system, calculates MRP by using ERP based on product BOM (Bill of Materials) information and inventory information, takes advantage of the MRP result to generate MPS. After customer order confirmation, pull production is driven by the final assemble schedule (FAS).

Product model series of tractor assembly enterprise is made up of $\mathrm{X}$ or $\mathrm{Y}$ two drive power series, 4WD power series, two drive high power series, 4WD high power series and $\mathrm{G}$ series. There are 2000 basic types. The daily maximum yield is 120 units. Enterprises have successfully implemented ERP and MES information projects, the planned throughput of MPS is 240 units. MPS as shown in table 1 . Through the MES platform, model type, delivery requirement, product model coding, quantity requirement, product BOM and material type code can be automatic acquisition. The question is how to schedule the optimization sorting of mixed-model assembly production within 3 days' planning date.

\begin{tabular}{cccc}
\hline Model code & Model & Quantity & Remarks \\
\hline 120206500065 & Y750 & 15 & \\
120207500209 & Y750A1 & 8 & Extra urgent \\
120208000163 & X900D71 & 17 & \\
120210000592 & X950A1 & 16 & \\
120211000264 & Y704A2Y0 & 5 & \\
120212000002 & Y754A4N1 & 13 & urgent \\
120212000007 & X754SA4Y0 & 18 & \\
120212000006 & Y804Y0 & 5 & \\
120213000002 & Y1200 & 14 & \\
120213000024 & X1200Y1 & 13 & \\
120407000532 & X1204 & 14 & \\
120408001924 & X1204K & 33 & \\
120409500705 & Y1304 & 17 & \\
120409500559 & G954E1N0Y0 & 17 & \\
120410000589 & G954E1K & 22 & \\
120411000688 & G954SK & 13 & \\
\hline & & & \\
\hline
\end{tabular}

Table 1 MPS 


\section{B Application of optimization algorithm}

Scheduling optimization algorithm--IOHA based on MES platform and Java programming language achieved artificial intelligence optimization scheduling. The instance set weights $\alpha=\beta=\gamma=\delta=0.5$, Tzzk $\varepsilon=0.1$, iteration times $\mathrm{N} \geq 10$, getting $\mathrm{N}$ scheduling $\left[T_{i}, q_{i t}\right]$. Feasible solution was searched which corresponds to $\min \mathrm{f}\left(T_{i}, q_{i t}\right)$ through adaption function $\mathrm{f}\left(T_{i}, q_{i t}\right)$. Eventually, assembly production scheduling plan was output automatically by MES platform, the result as shown in figure 3.

Figure 3: Output optimization result of assembly production scheduling plan

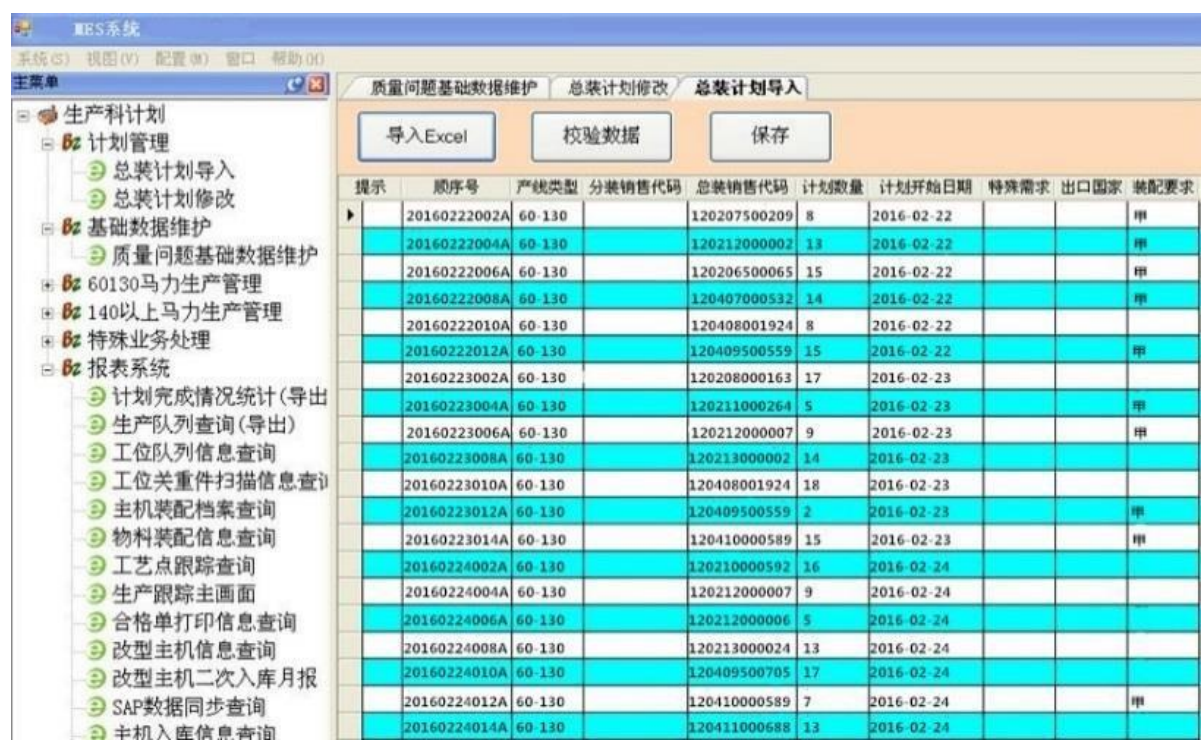

Assembly production scheduling plan which was optimized by artificial intelligence optimization not only could real-time sharing production instruction file, but also real-time change with inserting order, cancelling order and breakdown. This plan improved market response speed and the establishment efficiency of production planning, achieved the goal of lean production on time and balanced production.

The results show that the optimization method shortens the production preparation time by $67 \%$, identified and eliminated the bottleneck materials accurately, and $100 \%$ satisfied the customer requirements.

\section{VII.CONCLUSIONS}

The problem of SBC-MAP is a multidisciplinary and multi-objective complex optimization problem which depend on the traditional optimization methods can't solve the problems of complexity, lean and real-time. This paper proposed a multidisciplinary method which integrated mathematical programming, information technique, management technique, explored a novel SBC-MAP with high efficiency and real-time scheduling. Mathematical programming model of Multi-objective Collaborative Optimization (MCO) was constructed which based on the optimization target with market fast response and equilibrium production, regards the variation coefficient of delivery date, model and dispersion coefficient of daily yield equilibrium as KPI, takes bottleneck materials and throughput as the constraint. The IOHA of MS and AE was explored based on MES platform. Through finite cyclic iteration studied, we came to conclusion that regard the minimum scheme of fitness function as the feasible solution. This algorithm improved quality and efficiency, guaranteed the real-time and reliability of scheduling. Agricultural equipment production is a typical SBC-MAP model. Satisfactory results was obtained by taking tractor as the example to apply the research achievements in practices, not only shorten the production preparation cycle, improved efficiency, and realized balanced production, but could realize online accurate identification and removed bottleneck materials via MES platform. Meanwhile, production scheduling could be changed in real-time to meet customer requirements with rapid response. The results show that real-time optimization scheduling model and algorithm of SBC-MAP is effectiveness and feasibility and provides theoretical support for manufacturing to improve the market response rapid and lean production level.

\section{ACKNOWLEDGEMENTS}

This research is supported by the fund of China innovation method project (No. 2016IM030200), Henan universities science and technology innovation team, China (No. 15IRTSTHN008), Henan industry-university institute (IUI) project (No. 162107000030) and doctoral research foundation of Henan University of Science and Technology. Besides, the practical application support 
from China Tractor Group Co., Ltd is very important for this research.

\section{REFERENCES}

[1] L Wester, and M Kilbridge. 1963. The assembly line model-mix sequencing problem,Proceedings of the third international conference on Operations Research,pp 247-260

[2] Maurizio Faccio, Mauro Gamberi, and Marco Bortolini. 2016. Hierarchical approach for paced mixed-model assembly line balancing and sequencing with jolly operators,International journal of production research,54(3), pp 761-777.

[3] Hugejile Jo, Shuki Ge, Xue. 2016. A scheduling problem of the mixed model assembly line considering workload leveling in SPS picking area,The 13th International Conference on Industrial Management, Hiroshima, Japan,September 21-23, pp 92-96.

[4] Simon Emde. 2017. Scheduling the replenishment of just-in-time supermarkets in assembly plants, Or spectrum, 39(1),pp 321-345.

[5] Ibrahim Kucukkoc, David Z. Zhang. 2014. Mathematical model and agent based solution approach for the simultaneous balancing and sequencing of mixed-model parallel two-sided assembly lines,Int. J. Production Economics,158(2014), pp 314-333.

[6] Atan Z, De Kok T, and Dellaert NP. 2016. Setting Planned Lead times in Customer-Order-Driven Assembly Systems,M \& SOMMANUFACTURING \& SERVICE OPERATIONS MANAGEMENT, 18(1), pp 122-140.

[7] LU Jian-sha, WENG Yao-weil, and LI Xiu-lin et al. 2014. Application of hybrid artificial bee colony algorithm in mixed assembly lines sequencing,Computer Integrated Manufacturing Systems,20(1),pp 122-127.

[8] MENG Qiu-nan, ZHAO Cong, WANG Dan-ying, and YU Xiaoting. 2014. Mixed-flow Task Scheduling in No-wait Flowshop for General-sub Assembly,Industrial Engineering and Management,19(6),pp 29-36.

[9] Nils Boysen, Armin Scholl, Nico Wopperer. 2012. Resequencing of mixed-model assembly lines: survey and research agenda,European Journal of Operational Research, 216(3),pp 594604

[10] LIU Qiong, FAN Zheng-wei, ZHANG Chao-yong et al. 2014. Resequencing problem of mixed model assembly line caused by material unkitting,Computer Integrated Manufacturing Systems,20(7), pp 1608-1614.

[11] Hsiang-HsiHuang, WenPei, Horng-Huei Wu et al. 2013. A research on problems of mixed-line production and the rescheduling,Robotics and Computer-Integrated Manufacturing,29(2013),pp 64-72.

[12] Zemczak Marcin. 2014. Production Orders Sequencing in MixedModel Assembly Lines,ENGINEERING SOLUTIONS AND TECHNOLOGIES IN MANUFACTURING, 657(2014),pp 359363.
[13] Albert Corominas. 2016. A note on 'A bit-wise mutation algorithm for mixed-model sequencing in JIT production systems',International Journal of Production Research, 54(16),pp 5026-5027.

[14] Yu-Guang Zhong, Xiao-Xiao Lv, Zhan Yong. 2016. Sequencing problem for a hull mixed-model assembly line considering manufacturing complexity,Journal of Intelligent \& Fuzzy Systems,30(3), pp 1461-1473.

[15] Ibrahim Kucukkoc, David Z Zhang. 2016. Integrating ant colony and genetic algorithms in the balancing and scheduling of complex assembly lines,Int J Adv Manuf Technol, 82(1-4),pp 265-285.

[16] Jordi Pereira, Mariona Vila. 2015. An exact algorithm for the mixed-model level scheduling problem,International Journal of Production Research,53(19),pp 5809-5825.

[17] F. Tonelli, M. Paolucci, D. Anghinolfi, P. Taticchi. 2013. Production planning of mixed-model assembly lines: a heuristic mixed integer programming based approach,Production Planning \& Control,24(1),pp 110-127.

[18] Li XY, Qin K, Zeng, B, et al. 2016. Assembly sequence planning based on an improved harmony search algorithm,Int J Adv Manuf Technol,84(9-12),pp 2367-2380.

[19] ZHENG Yong-qian, WANG Yong-sheng. 2011. An Immunity Particle Swarm Sequencing Algorithm for Mixed-model Assembly Lines,Industrial Engineering and Management,16(4), pp 16-27.

[20] HAILI Lu, LONGFEI He, GEORGE Q Huang et al. 2016. Development and comparison of multiple genetic algorithms and heuristics for assembly production planning,IMA JOURNAL OF MANAGEMENT MATHEMATICS,27(2),pp 181-200.

[21] Wang Binggang, Rao Yunqing, Shao Xinyu, Xu Chi. 2009. A MOGA-based Algorithm for Sequencing a Mixed-mode Fabrication/Assembly System,China mechanical engineering,20(12),pp 1434-1438.

[22] Han Yudong, DONG Shangfei, TAN Baichuan. 2015. Multiobjective optimization for mixed-model assembly line balancing problem based on improved genetic algorithm,Computer Integrated Manufacturing Systems,(21)6, pp 1476-1485.

[23] Farzad Tahriri, Siti Zawiah Md Dawal, and Zahari Taha. 2014 Fuzzy Mixed Assembly Line Sequencing and Scheduling Optimization Model Using Multiobjective Dynamic Fuzzy GA,The Scientific World Journal, 2014, pp 1-20.

[24] MVA Raju Bahubalendruni, and Bibhuti Bhusan Biswal. 2016. A review on assembly sequence generation and its automation,PROCEEDINGS OF THE INSTITUTION OF MECHANICAL ENGINEERS PART C-JOURNAL OF MECHANICAL ENGINEERING SCIENCE,230(5),pp 824-838.

[25] Yang, Xiaoyin, Shi, Guohong,Zhang, Zhiwen. 2014. Collaboration of large equipment complete service under cloud manufacturing model,International Journal of Production Research,52(2),pp 326336 